\title{
The Journal of the Acoustical Society of America
}

HOME

BROWSE

MORE $\nabla$

Full

Published Online: April 2016

\section{Contribution of near- and suprathreshold hearing deficits to speech recognition}

Anna Warzybok, Sabrina Pieper, and Sarah Verhulst

View Affiliations

The Journal of the Acoustical Society of America 139, 1995 (2016); doi: http://dx.d oi.org/10.1121/1.4949849
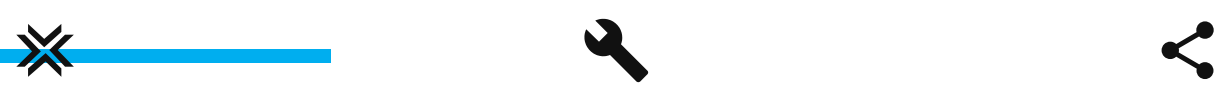

Il 


\section{ABSTRACT}

This study addresses the contribution of audibility and suprathreshold deficits to speech recognition in quiet, stationary, and speechmodulated noise. The relative importance of these deficits for different frequency ranges of hearing was determined by measuring speech recognition using broadband speech and noise, but also highand low-pass filtered versions of these stimuli. Audibility was assessed using pure tone thresholds and speech reception thresholds (SRT) in quiet. Three listener groups were considered to parse out hearing deficits: young $(\mathrm{yNH})$ and older normal-hearing $(\mathrm{oNH})$ listeners and hearing-impaired (HI) listeners. oNH and HI listeners performed equally poor in stationary noise where yNH listeners showed significantly better SRTs in both noise conditions. Since audibility did not correlate with SRTs in stationary noise but with modulated noise, and SRTs in stationary and modulated noise correlated with each other, a common mechanism (not audibility) must affect speech recognition in noise. Furthermore, our results suggest that performance in the broadband condition cannot be explained by the low frequency mechanisms since all groups performed similar in the low-pas conditions whereas the oNH and oHI group performed equally bad in high-pas condition. Instead, we found evidence for a contribution of high frequency envelope coding mechanisms to broadband performance 


\section{Resources}

AUTHOR

LIBRARIAN

ADVERTISER

\section{General Information}

ABOUT

CONTACT

HELP

PRIVACY POLICY

TERMS OF USE

FOLLOW AIP PUBLISHING:

f $y$ in 